\title{
SLIDING MODE PREDICTIVE CONTROL FOR CHEMICAL PROCESS WITH TIME DELAY
}

\author{
Winston García-Gabín* Darine Zambrano*E. F. Camacho**
}

\author{
* Universidad de Los Andes, Mérida 5101, Venezuela \\ Telf: +58 2742402802, Fax: +58 2742402890, \\ \{winstong,darine\}@ula.ve \\ ** Universidad de Sevilla, Sevilla 41092 España \\ Telf: +34 954487347, Fax: +34954487340 \\ eduardo@cartuja.us.es
}

\begin{abstract}
A design of a novel model predictive controller is presented. The proposed Sliding Mode Predictive Control (SMPC) algorithm combines the design technique of Sliding-Mode Control (SMC) with Model based Predictive Control (MPC). The SMPC showed a considerable robustness improvement with respect to MPC in the presence of time delay, and showed an enhanced ability to handle set point changes in a nonlinear process. Its robustness was evaluated using a robustness plot, its performance was judged using a single input single output nonlinear mixing tank process with variable time delay. Copyright (c)2005 IFAC
\end{abstract}

Keywords: Process control, Predictive control, Sliding-mode control, Time delay, Robustness.

\section{INTRODUCTION}

Time delay is a common phenomenon in industrial processes. It can be produced by the transport time required for a fluid to flow through a pipe or by the measurement and analysis lag. The presence of a time delay complicates significantly the analysis and the design of feedback controllers. It generally has awful consequences in the control loop performance, due to the introduction of an unstable behaviour, making more difficult to achieve a satisfactory control. Time delay limits the value of proportional controller gain at which the phase angle crosses $-180^{\circ}$ and the system became unstable, thus smaller controller gains are required as consequence sluggish response in a controlled variable is obtained (Ogunnaike and Ray, 1994). With a time delay the control action is based on delayed, hence obsolete, process information usually not representative of the current process output. Special care should be taken when the process time delay-time constant ratio is bigger than one, because PID controller can be inadequate, and therefore others control structures could be required. The control problem of time delay systems has received considerable attention over the last years (Richard, 2003),

Model Based Predictive Control (MPC) has become one of the most popular control methodologies for both industry and academia. It has been successfully implemented in many industrial applications, showing good performance. The basic idea of MPC is to calculate a sequence of future control signals in such a way that it minimizes a multistage cost function defined over a prediction horizon. The performance index to be optimised is the expectation of a quadratic function measuring the distance between the predictive system output and a predictive reference sequence over the horizon, plus a quadratic function measuring control effort. In order to implement an MPC, 
a model of the plant is used to predict the future plant outputs. This prediction is based on past and current values of the input and the output of the plant. Time delay is inherently considered in the prediction model of MPC. Thus, this control strategy is adequate for process with time delay. The advantages to apply MPC to time delay systems have been reported in literature (Maciejowski, 2001; Camacho and Bordóns, 1999; Levine, 1996; Soeterboek, 1991; Bitmead et al., 1990).

On the other hand, Sliding Mode Control (SMC) is a technique derived from Variable Structure Control (VSC) which was studied originally by Utkin (Utkin, 1981). For a broad class of systems, this kind of control is particularly appealing due to its ability to deal with nonlinearities, time-variance, as well as uncertainties and disturbances, in a direct manner in the face of modelling errors. In VSC, the control can modify its structure. The design problem consists of selecting the parameters of each structure and defining the traveling logic. The first step in SMC is to define a sliding surface, $S(t)$, along which the process can slide to find its desired final value. In general, the switching surface represents the system behaviour during the transient period, therefore, it must be designed to represent a desired system dynamics. The structure of the control system is intentionally altered as its state crosses the sliding surface in accordance with a prescribed control law. The second step is to design the control law in such a way that any state outside the sliding surface is driven to reach the surface in finite time and stay on it. SMC involving timedelay systems has received increasing attention (Gouaisbaut and Richard, 2002; Yuanqing and Yingmin, 2003; Niu Y. and X., 2004)

An algorithm based on variable structure control and generalized predictive control was proposed in (Corradini and Orlando, 1997). The sliding surface prediction was made only with past values of input and it did not consider the future control values. A dual mode control scheme combining nonlinear MPC and SMC was presented in (Zhou et al., 2001). MPC was used to force the state into a terminal region within a finite horizon while it is outside the terminal region and a sliding mode variable structure controller is used while the state is inside the terminal region.

The proposed single input single output controller is based on the idea of a combination of MPC and SMC that also has the future control movements for predicting the sliding surface, this results in more precise predictions and allows the process to be controlled with time delay; the other part of the control law is also simple and with fewer parameters and they have a clear meaning for tuning. The
SMPC is a single mode controller, the main idea is to introduce the prediction of the sliding surface into the control objective. SMPC has been applied to non minimum phase systems (García-Gabín and Camacho, 2003). It showed that an appropriate choice of the tuning parameters of SMPC avoids the instability problems of MPC when it is applied to non minimum phase systems. When MPC is used the controller achieves the optimal output by cancelling the plant zeros, including the unstable zeros, which leads to a loss of internal stability of the feedback system. SMPC shows a considerable robustness improvement with respect to MPC in the presence of modelling uncertainties and disturbances, while enhanced its ability to handle set point changes in a nonlinear process with inverse response.

This article is organized as follows: Section 2 presents the development of SMPC. Section 3 shows the controller robustness. Section 4 shows the application SMPC for a mixing tank. This is a nonlinear process with variable time delay. Finally, the conclusions are presented.

\section{SLIDING MODE PREDICTIVE CONTROL}

Most SISO plants when considering operation around a particular set-point and after linearization can be described by:

$A\left(z^{-1}\right) y(t)=z^{-d} B\left(z^{-1}\right) u(t-1)+C\left(z^{-1}\right) \frac{\xi(t)}{\Delta}(1)$

Where: $y(t)$ is the process output signal, $u(t)$ is the process input signal, $\Delta: 1-z^{-1}, d:$ is the delay, $\xi(t)$ is the zero mean white noise, $A\left(z^{-1}\right)$ and $C\left(z^{-1}\right)$ are monic polynomials, and $B\left(z^{-1}\right)$ is a polynomial that has the zeros of the model. This model is known as the CARIMA Model (Controller Auto-Regressive Integrated MovingAverage). This model can be used for openloop stable, open-loop unstable, integrating, or nonminimum phase systems It has been argued that for many industrial applications in which disturbances are non-stationary CARIMA model is more appropriate (Clarke et al., 1987). The most usual case $\mathbf{C}\left(z^{-1}\right)=1$ has been used because in practice the colouring polynomial are very difficult to estimate with sufficient accuracy (Camacho and Bordóns, 1999).

The following new predictive sliding surface is proposed to develop the controller:

$$
\begin{aligned}
S_{t+j \mid t}= & P_{s}\left(z^{-1}\right)(y(t+j \mid t)-w(t+j))+ \\
& Q_{s}\left(z^{-1}\right) \Delta u_{C}(t+j-1-d)
\end{aligned}
$$

where $w(t)$ is trajectory reference, $P_{s}\left(z^{-1}\right)$ and $Q_{s}\left(z^{-1}\right)$ are polynomials of degree $n p$ and $n q$ respectively, given by, 


$$
\begin{aligned}
P_{s}\left(z^{-1}\right) & =p_{s_{0}}+p_{s_{1}} z^{-1}+\ldots+p_{s_{n p}} z^{-n p} \\
Q_{s}\left(z^{-1}\right) & =q_{s_{0}}+q_{s_{1}} z^{-1}+\ldots+q_{s_{n q}} z^{-n q}
\end{aligned}
$$

The switching surface represents the system behaviour during the transient period, therefore, it must be designed to represent a desired system dynamics. Small roots of $P_{s}\left(z^{-1}\right)$ produce a fast dynamic in the sliding surface.

A predictive sliding surface is also presented in (Corradini and Orlando, 1997), however, it does not use the future control signals $\Delta u(t-1+j)$ to predict the future sliding surface values $S_{t+j \mid t}$, as is done here. Notice that using the future control deviations allows better predictions of the future values of the sliding surface, especially for control process with time delay. The general aim is that the future predictive surface (2) on the considered horizon should be zero and at the same time, the control effort $\Delta u_{C}$ necessary for doing so should be penalized. The expression for the objective function is given by,

$$
\begin{aligned}
J= & \sum_{j=N_{1}}^{N_{2}}[\hat{S}(t+j \mid t)]^{2}+ \\
& \sum_{j=1}^{N_{u}} \lambda(j)\left[\Delta u_{C}(t+j-1)\right]^{2}
\end{aligned}
$$

where $\hat{S}(t+j \mid t)$ is an optimum $j$-step prediction of the sliding surface on data up to time $t, N_{1}$ and $N_{2}$ are the minimum and maximum predictive horizons, $N_{u}$ is the control horizon, and $\lambda(j)$ are weighting sequences. The objective of the controller is to compute the future control sequence in such way that the future surface $S(t+j)$ is driven close to zero. The minimization of the objective function $J\left(N_{1}, N_{2}, N_{u}\right)$ produces $\Delta u_{C}(t), \Delta u_{C}(t+$ $1), \cdots, \Delta u_{C}\left(t+N_{u}\right)$. The final objective of control is to ensure that the controlled variable is close to its reference value $w(t+j)$ at all times, meaning that $e(t)$ must be zero. The problem of tracking a reference value can be reduced to keeping $S(t)$ at zero. The closed-loop dynamic is chosen with the parameters of polynomials $P_{s}\left(z^{-1}\right)$ and $Q_{s}\left(z^{-1}\right)$. Once the sliding surface has been selected, attention must be turned to designing the control law that satisfies $S(t)=0$. The control law, $\Delta u(t)$, consists of two additive parts, $\Delta u_{C}(t)$, and $\Delta u_{D}(t)$. That is,

$$
\Delta u(t)=\Delta u_{C}(t)+\Delta u_{D}(t)
$$

The control signal $\Delta u_{C}(t)$ is given by a Model Based Predictive Control algorithm using (5). $u_{D}(t)$, incorporates a nonlinear predictive element that includes the switching element of the control law. This control sequence is given by,

$$
u_{D}(t+j)=K_{D} \frac{S(t+j \mid t)}{|S(t+j \mid t)|+\rho}
$$

where $K_{D}$ is a gain which is the tuning parameter responsible for the reaching mode, and $\rho$ is a tuning parameter used to reduce the chattering problem (Zinober, 1994). In order to minimize (5), the $j$-step ahead output prediction $\hat{S}(t+j \mid t)$ for $j=N_{1}, \cdots, N_{2}$ has been computed based on the information known at time $t$ and the future values of the control increments. The following Diophantine equation is considered,

$$
1=E_{j}\left(z^{-1}\right) \tilde{A}\left(z^{-1}\right)+z^{-j} F_{j}\left(z^{-1}\right)
$$

The polynomial $E_{j}\left(z^{-1}\right)$ and $F_{j}\left(z^{-1}\right)$ are uniquely defined with degrees $j-1$ and na respectively, $\tilde{A}\left(z^{-1}\right)=\Delta A\left(z^{-1}\right)$. Using the plant model and a Diophantine equation the follow prediction output equation can be obtained,

$$
\begin{aligned}
\hat{y}(t+j)= & E_{j}\left(z^{-1}\right) B\left(z^{-1}\right) \Delta u_{C}(t+j-1-d) \\
& +F_{j}\left(z^{-1}\right) y(t)
\end{aligned}
$$

In this expression $\hat{y}(t+j)$ is a function of a known signal value at time $t$ and also of future control inputs which have not yet been computed . Using a second Diophantine equation (10) to distinguish past and future control values,

$$
E_{j}\left(z^{-1}\right) B\left(z^{-1}\right)=G_{j}\left(z^{-1}\right)+z^{-j} \Gamma_{j}\left(z^{-1}\right)
$$

The polynomial $G_{j}$ contains the first $j$ step response parameters of the plant model. The following expression of the prediction is obtained,

$$
\begin{aligned}
\hat{y}(t+j)= & G_{j}\left(z^{-1}\right) \Delta u_{C}(t+j-1-d) \\
& +\hat{y}(t+j \mid t)
\end{aligned}
$$

where $\hat{y}(t+j \mid t)$ is the free response prediction of $\hat{y}(t+j)$ assuming that future control increments after time $t-1$ will be zero,

$$
\begin{aligned}
\hat{y}(t+j \mid t)= & \Gamma_{j}\left(z^{-1}\right) \Delta u_{C}(t-1-d) \\
& +F_{j}\left(z^{-1}\right) y(t)
\end{aligned}
$$

Substituting $E_{j}\left(z^{-1}\right)$ of (8) into (10), this yields

$$
\begin{aligned}
B\left(z^{-1}\right)= & z^{-j} \tilde{A}\left(z^{-1}\right) \Gamma_{j}\left(z^{-1}\right)+z^{-j} F_{j}\left(z^{-1}\right) B\left(z^{-1}\right) \\
& +\tilde{A}\left(z^{-1}\right) G_{j}\left(z^{-1}\right)
\end{aligned}
$$

Define the vector $f(t)$, composed of the free response predictions,

$$
f(t)=\left[\hat{y}(t+1+d \mid t), \cdots, \hat{y}\left(t+N_{2}+d \mid t\right)\right]^{T}(14)
$$

the vector of future control increments,

$$
\Delta u_{C}(t)=\left[\Delta u_{C}(t), \cdots, \Delta u_{C}\left(t+N_{u}-1\right)\right]^{T}
$$

From prediction (11) the predicted input-output relationship of the plant can be written as,

$$
\hat{y}(t)=\mathbf{G} \Delta u_{C}(t)+f(t)
$$


where matrix $\mathbf{G}$ is composed of $g_{k}$ step response parameters of the SISO plant model.

$$
\mathbf{G}=\left[\begin{array}{cccc}
g_{0} & 0 & \cdots & 0 \\
g_{1} & g_{0} & \cdots & 0 \\
\vdots & \vdots & \ddots & \vdots \\
g_{N_{2}-1} & g_{N_{2}-2} & \cdots & g_{N_{2}-N_{u}}
\end{array}\right]
$$

The prediction of the sliding surface is obtained substituting (16) into (2),

$\hat{S}(t)=\left(\mathbf{P}_{\mathbf{s}} \mathbf{G}+\mathbf{Q}_{\mathbf{s}}\right) \Delta u_{C}(t)+\mathbf{P}_{\mathbf{s}}\left(f_{s}(t)-w(t)\right)(18)$

Where the free response of the sliding surface $f_{s}$ is given by,

$$
\begin{aligned}
f_{s}(t)= & \mathbf{F}\left(z^{-1}\right) y(t)+\Gamma\left(z^{-1}\right) \Delta u_{C}(t-1)+ \\
& \mathbf{P}_{\mathbf{s}}{ }^{-1} \mathbf{P}_{\mathbf{s}}{ }^{*} e(t)+\mathbf{P}_{\mathbf{s}}{ }^{-1} \mathbf{Q}_{\mathbf{s}}{ }^{*} \Delta u_{C}(t-1)
\end{aligned}
$$

with the matrices defined as,

$$
\begin{gathered}
\mathbf{P}_{\mathbf{s}}=\left[\begin{array}{cccc}
p_{s 0} & 0 & \cdots & 0 \\
p_{s 1} & p_{s 0} & \cdots & 0 \\
\vdots & \vdots & \ddots & \vdots \\
0 & p_{s_{n p}} & \cdots & p_{s 0}
\end{array}\right] \\
\mathbf{P}_{\mathbf{s}}^{*}=\left[\begin{array}{cccc}
p_{s 1} & \cdots & p_{s_{n p-1}} & p_{s_{n p}} \\
p_{s 2} & \cdots & p_{s_{n p}} & 0 \\
\vdots & \vdots & \vdots & \ddots \\
0 & 0 & \cdots & 0
\end{array}\right] \\
\mathbf{Q}_{\mathbf{s}}=\left[\begin{array}{cccc}
q_{s 0} & 0 & \cdots & 0 \\
q_{s 1} & q_{s 0} & \cdots & 0 \\
\vdots & \vdots & \ddots & \vdots \\
0 & q_{s_{n q}} & \cdots & q_{s 0}
\end{array}\right] \\
\mathbf{Q}_{\mathbf{s}}^{*}=\left[\begin{array}{cccc}
q_{s 1} & \cdots & q_{s_{n q-1}} & q_{s_{n q}} \\
q_{s 2} & \cdots & q_{s_{n q}} & 0 \\
\vdots & \vdots & \vdots & \ddots \\
0 & 0 & \cdots & 0
\end{array}\right]
\end{gathered}
$$

The objective function (5) can be rewritten as,

$$
\begin{aligned}
J= & \sum_{j=N_{1}}^{N_{2}}\left[\mathbf{P}_{\mathbf{s}} \mathbf{G} u_{C}+\mathbf{P}_{\mathbf{s}}\left(f+\mathbf{P}_{\mathbf{s}}^{*}+\mathbf{Q}_{\mathbf{s}}^{*} u_{C}\right)-\mathbf{P}_{\mathbf{s}} w\right. \\
& \left.+\mathbf{Q}_{\mathbf{s}} u_{C}\right]^{2}+\sum_{j=1}^{N_{u}} \lambda(j)\left[\Delta u_{C}(t+j-1)\right]^{2}
\end{aligned}
$$

The quadratic minimization of (24) becomes a direct problem of linear algebra, assuming there are no constraints on the control signal, which leads to,

$$
\begin{aligned}
\Delta u_{C}(t)= & K_{S M P C}\left(w(t)-f_{s}(t)\right) \\
K_{S M P C}= & {\left[\left(\mathbf{P}_{\mathbf{s}} \mathbf{G}+\mathbf{Q}_{\mathbf{s}}\right)^{T}\left(\mathbf{P}_{\mathbf{s}} \mathbf{G}+\mathbf{Q}_{\mathbf{s}}\right)+\lambda I\right]^{-1} } \\
& \left(\mathbf{P}_{\mathbf{s}} \mathbf{G}+\mathbf{Q}_{\mathbf{s}}\right)^{T} \mathbf{P}_{\mathbf{s}}
\end{aligned}
$$

and

$$
\begin{aligned}
\Delta u_{D}(t)= & {\left[\frac{K_{D} \hat{S}(t+d)}{|\hat{S}(t+d)|+\rho}, \cdots\right.} \\
& \left.\frac{K_{D} \hat{S}\left(t+N_{u}+d-1\right)}{\left|\hat{S}\left(t+N_{u}+d-1\right)\right|+\rho}\right]
\end{aligned}
$$

finally, the control signal is given by,

$$
\Delta u(t)=K_{S M P C}\left(w(t)-f_{s}(t)\right)+\Delta u_{D}(t)
$$

To summarize, the SMPC has two parts. $\Delta u_{D}(t)$, and responsible for guiding the system to the sliding surface, and $\Delta u_{C}(t)$ developed like an MPC, which is responsible for keeping the controlled variable on the reference value. Note that choosing $P_{s}\left(z^{-1}\right)=1, Q_{s}\left(z^{-1}\right)=0$, the objective function is reduced

$$
\begin{aligned}
J= & \sum_{j=N_{1}}^{N_{2}}[\hat{y}(t+j \mid t)-w(t+j)]^{2}+ \\
& \sum_{j=1}^{N_{u}} \lambda(j)\left[\Delta u_{C}(t+j-1)\right]^{2}
\end{aligned}
$$

and the $K$ matrix gain is the usual linear MPC,

$$
K_{M P C}=\left(\mathbf{G}^{T} \mathbf{G}+\lambda I\right)^{-1} \mathbf{G}^{T}
$$

\section{CONTROLLER ROBUSTNESS}

This section compared the robustness of the controller proposed against a MPC. This is evaluated using a robustness plot (Shinskey, 1990). In the robustness plot the stability limit for a closed loop is plotted on logarithmic coordinates when the process time delay and gain change. The axes of this plot are the delay ratio (the process time delay over the process time delay the controller was tuned) and the gain ratio (the process gain over the process gain the controller was tuned). All combination of delay ratio and gain ratio within a factor of two in either direction are enclosed within a robustness window. If the stability limit for a closed loop stays outside of this window, that loop is considered to be robust. To plot the stability limit, a controller is first tuned for a base set of process parameters, represented by the middle point $(1,1)$ on the coordinates. The process time delay is then changed in ratio to the base time delay until uniform oscillations are produced; this is the delay ratio at the limit of stability. Similarly, the process gain is changed in ratio to the base gain until the limit of stability can be reached. Or both can be changed simultaneously. The locus of such points represents the stability envelope for that particular combination of process, controller and tuning.

The robustness plot was constructed initially tuning both controllers to have similar settling time 


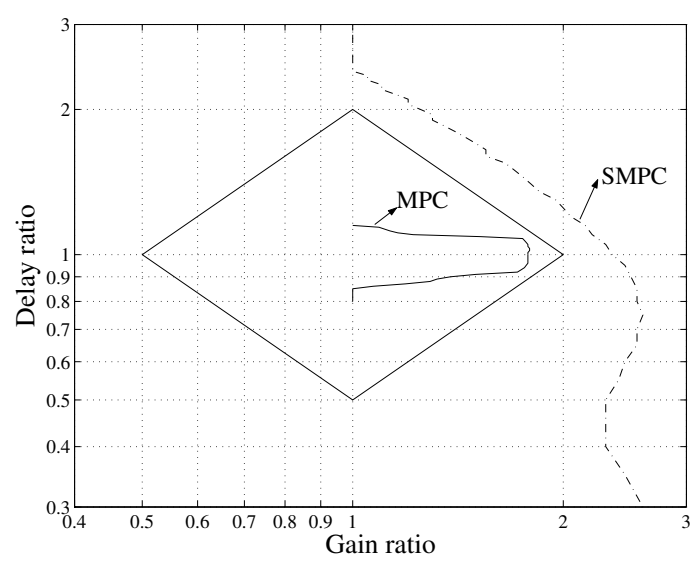

Fig. 1. Robustness plot

using the follows model, ( Note that controllability ratio is one $\left.\left(\frac{t_{o}}{\tau}=1\right)\right)$

$$
G_{p}=\frac{1}{s+1} e^{-1 s}
$$

From Figure 1 the following values of Robustness Index $(\mathrm{RI})$ were obtained $(\mathrm{MPC}=0.78$ and $\mathrm{SMPC}=1.41)$. The results shown in this section demonstrated that the proposed SMPC has a more robust behaviour than the MPC.

\section{SIMULATION EXAMPLE}

In order to compare the SMPC and MPC performances a nonlinear model of a mixing tank process (Camacho and Smith, 2000), has been considered. the mixing tank receives two streams: a hot stream, $W_{1}(t)$, and a cold stream $W_{2}(t)$ (Figure 2). The outlet temperature is measured at a point $125 \mathrm{ft}$ downstream from the tank, this produces a variable time delay. The following assumptions are taken: The liquid volume in the tank is considered constant, the tank contents are well mixed, the tank and the pipe are well insulated. The temperature transmitter is calibrated for a range of 100 to $200^{\circ} \mathrm{F}$.

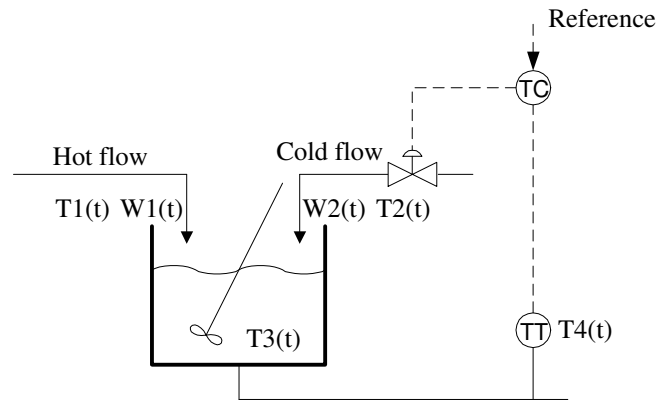

Fig. 2. Mixing tank

The SMPC and MPC were tuned initially to have similar settling time when perturbations around the operating point were simulated. The following tuning parameters were obtained: MPC: $N_{1}=40$; $N_{2}=90 ; N_{u}=25$ у $\lambda=20$, SMPC: $N_{1}=40 ;$ $N_{2}=90 ; N_{u}=20 ; \lambda=2 ; P_{s}=1-0.9 z^{-1} ; Q_{s}=$ $0.1 z^{-1} ; K_{D}=-0.2$ у $\rho=0.3$, both predictive controllers use the same prediction model:

$$
G_{p}=\frac{-0.95}{2.52 s+1} e^{-4 s}
$$

In this operating point the process has a controllability ratio bigger than one $\left(\frac{t_{o}}{\tau}=1.6\right)$. It is well known that this produces control difficulties (Smith and Corripio, 1997).

Figure 3 shows time delay changes when the hot stream was modified. The flow of hot water changes from $250 \mathrm{lb} / \mathrm{min}$ to $225 \mathrm{lb} / \mathrm{min}$, then to $200 \mathrm{lb} / \mathrm{min}$, and finally to $175 \mathrm{lb} / \mathrm{min}$. Time delay was increased a $20 \%$ with these variations of the hot stream.
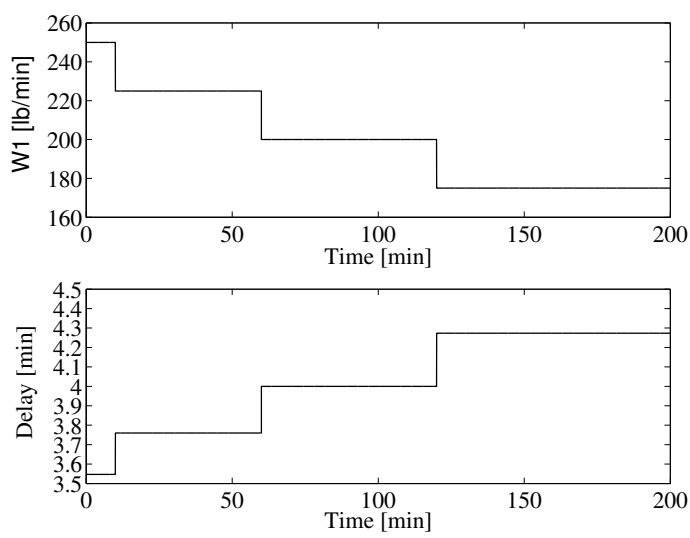

Fig. 3. Time delay changes vs hot stream variations $W_{1}(t)$

The response of the temperature (controlled variable) is shown in Figure 4. The output time courses clearly shown that as the operating conditions change, the performance of the MPC controller degraded, while the SMPC maintains its performance and stability. In this case, as the flow of hot water decreases, with a corresponding decrease in cold water, the time delay between the tank and the temperature sensor increases. This increment in time delay certainly adversely affects the performance of the MPC controller. In the Figure 5 the proposed controller is compared with a standard PI plus Smith Predictor $(\mathrm{PI}+\mathrm{SP})$, both controller have been tunning initially with the same settling time. When time-delay was increased SMPC shows better performance that $\mathrm{PI}+\mathrm{SP}$, also SMPC can use the future setpoint knowledge and can incorporate constraints.

\section{CONCLUSIONS}

The proposed single input single output SMPC algorithm combines the design technique of SMC 


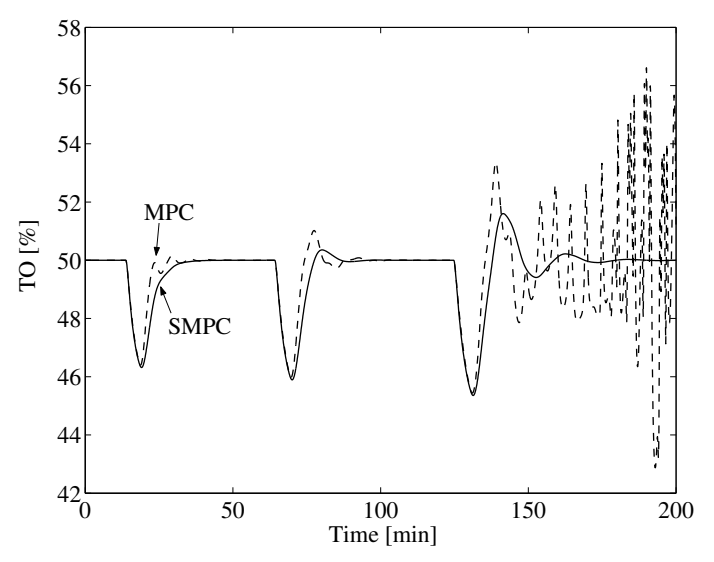

Fig. 4. SMPC and MPC performance for $W_{1}(t)$ changes

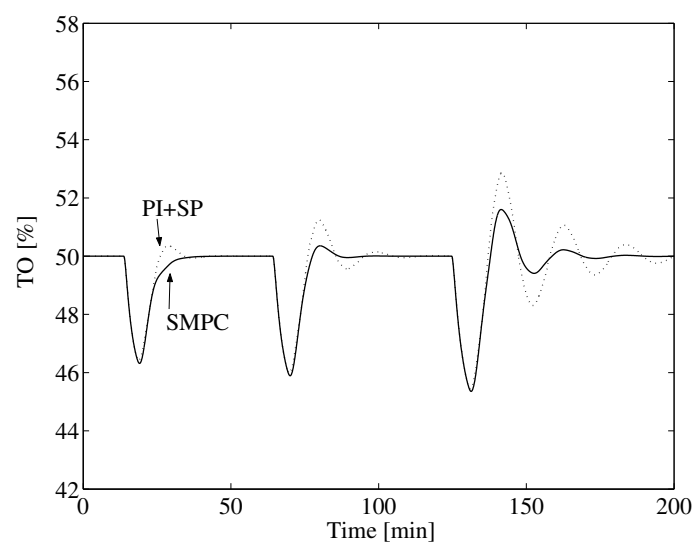

Fig. 5. SMPC and PI+SP performance for $W_{1}(t)$ changes

and MPC. It is shown that mixing both control techniques results in a new predictive control with a better robustness than a classic MPC. The robustness index quantitatively demonstrated that $\mathrm{SMPC}$ is more robust than classic MPC. The controller performance using a nonlinear process with variable time delay shows a considerable improvement in robustness with respect to MPC in the presence of modelling uncertainties, and disturbances and enhanced its ability for handling set point changes. The SMPC acquires the main advantages of the two control methods, the robustness features of sliding mode control and the good performance of model predictive control. The SMPC improves the closed loop behaviour of MPC avoiding the strong control movements of SMC. The computational requirements of SMPC are similar to those needed for MPC. It does not require more powerful hardware to be applied in whatever process where a classic MPC is being applied.

\section{REFERENCES}

Bitmead, R.R., M. Gevers and V. Wertz (1990). Adaptative Optimal Control: The Thinking man's GPC. Prentice Hall. Brunswick.
Camacho, E.F. and C. Bordóns (1999). Model Predictive Control. 2 ed.. Springer-Verlag. London.

Camacho, O. and C. Smith (2000). Sliding mode control an approach to regulate nonlinear chemical proceses. ISA Transaction 39(2), 205-218.

Clarke, D. W., C. Mohtadi and P. S. Tuffs (1987). Generalized Predictive Control: Part I: The Basic Algorithm. Automatica 23(2), 137-148.

Corradini, M. Letizia and G. Orlando (1997). A VSC algorithm based on generalized predictive control. Automatica 33(5), 927-932.

García-Gabín, W. and E.F. Camacho (2003). Sliding mode model based predictive control for non minimum phase systems. In: European Control Conference. Cambridge,UK.

Gouaisbaut, F., Dambrine M. and J.P. Richard (2002). Robust control of delay systems: a sliding-mode control design via lmi. Syst. Control Lett. 46(4), 219-230.

Levine, W.S. (Ed) (1996). The Control Handbook. IEEE Press. Boca de Ratón.

Maciejowski, J.M. (2001). Predictive Control with Constraints. Prentice Hall. Harlow.

Niu Y., Lam J. and Wang X. (2004). Robust sliding-mode control for uncertain time-delay systems: An lmi approach. IEE Proc.-Control Theory Appl. 151(1), 38-44.

Ogunnaike, B.A. and W.H. Ray (1994). Process Dynamics, Modeling, and Control. Oxford University Press. Oxford.

Richard, J.P. (2003). Time-delay systems:an overview of some recent advances and open problems. Automatica 39, 1667-1694.

Shinskey, F. G. (1990). How good are our controllers in absolute performance and robustness?. Measurement and Control 23, 114-121.

Smith, Carlos A. and Armando B. Corripio (1997). Principles and practice of automatic process control. 2 ed.. John Wiley \& Sons. USA.

Soeterboek, R. (1991). Predictive Control: A Unified Approach. Prentice Hall. New York.

Utkin, V. I. (1981). Sliding modes in control and optimization. Spring-Verlag. Moscow.

Yuanqing, X. and J. Yingmin (2003). Robust sliding-mode control for uncertain time-delay systems: An lmi approach. IEEE Transactions on Automatic Control 48(6), 10861092.

Zhou, J., Z. Liu and R. Pei (2001). A new non linear model predictive control scheme for discrete time systems based on sliding mode control. In: American Control Conference. Arlington. pp. 3079-3084.

Zinober, A.S. (1994). Variable Structure and Lyapunov Control. Spring-Verlag. London. 